
\title{
3 Research Square \\ MFGE8 Improved Brain Functional Connectivity Between CA1 and LEnt By Activating Efferocytosis in Hippocampus of SAE Rats
}

\section{Haojia Li}

Nanjing First Hospital Nanjing Medical University

Hongsen Liao

Nanjing First Hospital Nanjing Medical University

Jiayue Du

Nanjing First Hospital Nanjing Medical University

Xiaomin Xu

Nanjing First Hospital Nanjing Medical University

Yuchen Chen

Nanjing First Hospital Nanjing Medical University

Zhiyuan Zhang

Nanjing Medical University

Yanna Si

Nanjing First Hospital Nanjing Medical University

Hongguang Bao ( $\nabla$ hongguang_bao@hotmail.com )

Nanjing First Hospital Nanjing Medical University

\section{Research Article}

Keywords: Sepsis-associated encephalopathy (SAE), Milk fat globule EGF factor 8 protein (MFG-E8), Resting state functional magnetic resonance imaging (rs-fMRI), Efferocytosis, Microglia, Functional connectivity (FC)

Posted Date: January 20th, 2022

DOI: https://doi.org/10.21203/rs.3.rs-1264779/v1

License: (c) (i) This work is licensed under a Creative Commons Attribution 4.0 International License. Read Full License 


\section{Abstract}

Background: Sepsis-associated encephalopathy (SAE) is one of the most serious complications of sepsis, along with brain function and cognitive behavioral changes. The process of efferocytosis with microglia was restrained in SAE rats' hippocampus. We have previously shown that milk fat globule EGF factor 8 protein (MFG-E8) evoked efferocytosis in SAE rats' brain, and alleviated the inflammnation in hippocampus. The present study investigated the the contribution of MFG-E8 to the brain functional connectivity $(\mathrm{FC})$ of hippocampus and lateral entorhinal cortex in SAE rats by resting-state functional MRI scan.

Methods: Sixty adult male Sprague Dawley rats were divided randomly into Sham operation group, cecal ligation and puncture (CLP) group, CLP+MFGE8 group, and CLP+MFGE8+Cilengitide group. After cecal ligation and puncture (CLP) surgery, MFGE8 (3.3ug) was injected intracerebroventricularly (i.c.v.) and cilengitide (10 mg/kg) was injected intraperitoneal. The Morris water maze (MWM) and open filed test (OFT) were performed continuously for five days. Rats were detected by T2-MRI and rs-fMRI scan 8 days post-surgery, then brain tissues were taken for western blotting and pathological observation by HE stained. One-way ANOVA with Tukey's post-test of all pair of columns was employed statistical analyses.

Results: MFGE8 improved performance of neurobehavioral tests in MWM test and OFT. Expression of IL$1 \beta$ and the proteins of MFGE8, Rac1, and LC3 were up regulated in CLP+MFGE8 group. On the contrary, expression of IL-6, Rac1, and LC3 were down regulated in CLP+MFGE8+cilengitide group. MFGE8 significantly decreased the hyperintense region in hippocampus detected by T2-MRI, and increased ALFF value in SAE rats' bilateral sensory cortex, motor cortex and visual cortex by rs-fMRI. In addition, MFGE8 increased FC of CA1 and LEnt in same side of cerebral hemisphere, and the FC of CA1 and LEnt was consistent with the behavior result in Morris water maze test.

Conclusions: MFGE8 activated efferocytosis with microglia and improved brain function and functional connectivity between CA1 and LEnt which were reflected in behavior tests and fMRI.

\section{Background}

Sepsis is life-threatening organ dysfunction due to infection, causing about 11.0 million people death worldwide in 2017[1] . Sepsis-associated encephalopathy (SAE) is one of the most serious complication of sepsis which was defined as diffuse cerebral dysfunction with short of clinical evidence of direct brain infection. More than $50 \%$ of surviving patients with sepsis suffered from severe and long-term cognitive deficits [2]. In general, neuroinflammation, neurotoxicity, and apoptosis have been considered to be causative factors for cognitive impairment in SAE patients. In the process of SAE, the elimination of apoptosis cells and the secretion of inflammation factors relied on the normal function of microglia. However, the ability of microglia efferocytosis was deteriorated in SAE, and the precise mechanisms remain unknown. Furthermore, whether the activator of integrin receptor on microglia has protective effects on SAE brain injury need to be further researched. 
In pathological mechanism of sepsis associated encephalopathy, it is well known that the activation of microglia plays important roles for both pro-inflammation and anti-inflammation. Activated microglia release pro-inflammatory cytokines to damage neurons, meanwhile, they also scavenge damaged cells in central nervous system(CNS). Microglial phagocytosis maintained the homeostasis of CNS and guarantee the normal operating of neural network [3]. The process of efferocytosis, which is a form of microglial phagocytosis, involves four phases: apoptotic cell (AC) finding, AC binding, AC internalization and $A C$ degradation. In the second phases, microglial engage ACs through binding bridge molecules that interact with phosphoryl serine (PS) on the AC surface [4]. In many binding bridge molecules, Milk fat globule EGF factor 8 protein (MFG-E8) plays a crucial part in efferocytosis, which was secreted by Microglia and astrocytes. MFG-E8 consists of four domains, E1 and E2 homologous regions to epidermal growth factor (EGF), while $\mathrm{C} 1$ and $\mathrm{C} 2$ homologous regions to coagulation factor 8[5]. E2 region of MFGE8 attached the $\mathrm{aV} \beta 3$ and $\mathrm{aV} \beta 5$ integrins on the microglial surface, thereby, macrophage can recognize and uptake the apoptotic cells. After MFGE8 Combined with the $\mathrm{aV} \beta 3$ and $\mathrm{aV} \beta 5$ integrin receptors on the microglia surface, downstream molecule of Rac1 was regulated in the process of cytoskeletal reorganization and migration. The recent research found that rhMFG-E8 reduced the number of apoptotic cells and improved the 10-day sepsis survival rate of sepsis rats[6]. However, these past studies have been focused on systematic inflammation response in sepsis, without concentration on sepsis associated encephalopathy. Whether it can improve the brain function of SAE rats needs to be confirmed.

Presently, the estimation of brain function and diagnosis of SAE patients rely on questionnaire score and clinical symptoms including agitation, lack of focus, somnolence, and coma. The Glasgow Coma Scale and the Confusion Assessment Method (CAM) are helpful but not sensitive enough to detect focal neurological signs. Some technical examinations, such as computer tomography (CT) and magnetic resonance imaging (MRI), are often used to rule out intracranial bleeding and other conditions like brain infarction or abscesses[7]. Similarly, cognitive impairment occurred in the rodent models with sepsis induced by cecal ligation and puncture (CLP) or injection of lipopolysaccharide (LPS). The behavioral tests such as open field task, novel object recognition task, and Morris water maze test can reflect the cognitional deficient in SAE rats[8]. The pathological research confirmed that major lesions in SAE rats were impairment of the blood brain barrier (BBB), microglial activation and a decrease of neuronal densities in the cortex and the hippocampal formation. However, the noninvasive methods in evaluating brain function and progression of SAE were less applied, and the relationship between behavior and image changes needs further research.

Functional magnetic resonance imaging (fMRI) provides a tool for immediately detecting the active brain regions and evaluating the function of each brain area. This technique has been used to detect the changes of several diseases such as stroke, epilepsy, schizophrenia[9] and different state like rest stating, reminiscence, and anesthesia [10]. However, the research of evaluation therapy in sepsis associated encephalopathy patients by fMRI was limited. The patients with sepsis always suffered from severe multiple organ failure, and it was inconveniencing for them to finish the resting state MRI examination. By contrast, sepsis rats can be fixed well by inhaling $2 \%$ isoflurane during the MRI scan, therefore, they were suitable for cerebral function research by rs-fMRI[11]. Blood oxygenation level dependent (BOLD) fMRI 
detects changes of blood oxygen which was related to neuronal activity. The most successfully applied analysis methods of BOLD signal are amplitude of the low-frequency fluctuations (ALFF). ALFF measured the amplitude of the regional spontaneous neuronal activity[12]. And this method may provide a more comprehensive assessment of brain dysfunction. In addition, brain functional connectivity between different brain regions also can reflect the function coherence in some task or rest stating. However, few prior studies on the relationship between FC and behavioral abnormalities in SAE rats have been reported.

As we know, cecal ligation and puncture (CLP) surgery induced sepsis associated encephalopathy rats suffered hippocampus related cognitive impairment[13]. In the present study, we examined whether milk fat globule EGF factor 8 protein (MFG-E8) improve the brain function by fMRI and behavioristics in SAE rats.

\section{Methods}

\section{Animals and treatments}

All animal procedures were approved by the Institutional Animal Care and Use Committee of Nanjing Medical University(IACUC: 2103051), and the Number of Animal Use Permit is SYXK(Su)2020-0022. Totally 60 adult male Sprague Dawley rats (Jiangsu Laboratory Animal Center, China; 8 weeks, 280-320g) were housed under a $12 / 12 \mathrm{~h}$ light-dark cycle at $22-24^{\circ} \mathrm{C}$ with $50 \%-60 \%$ humidity. Food and water were available ad libitum.

Our animals experiment included four groups: Sham operation group $(n=15), \operatorname{CLP}$ group $(n=15)$, CLP+MFGE8 group $(n=15)$, and CLP+MFGE8+Cilengitide group $(n=15)$. Totally 3.3ug MFGE8 (LMAI Bio, ICA286Hu01) was injected intracerebroventricularly (i.c.v.) on the first day after CLP surgery for rats in CLP+MFGE8 group and CLP+MFGE8+Cilengitide group using a microsyringe with stereotaxic coordinates of AP-1.0 mm, ML2.0 mm, DV3.8 mm[14]. Cilengitide (MCE, HY-16141), inhibitor of aVB3 and aVß5 integrins receptor [15], was diluted with normal saline (10 mg/kg, $1 \mathrm{ml} /$ animal), and injected intraperitoneal one hour after CLP surgery for rats in CLP+MFGE8+Cilengitide group[16]. The rats were weighed every day during the experiment. Three days after CLP, the neurological behavior tests were measured continuously for five days. The Morris water maze (MWM) and open filed test (OFT) were performed on third day post-surgery. After behavioral observation finished, the animals were observed by fMRI scan to detect the brain changes of structure and function. And then, the rats were sacrificed, and the brain as well as serum were collected for proteins analysis and pathological observation.

\section{Cecal ligation and puncture}

Sepsis-associated encephalopathy model were induced by cecal ligation and puncture (CLP) surgery in male Sprague Dawley rats. After fasting for 6 hours, the rats were anesthetized by intraperitoneal injection $35 \mathrm{mg} / \mathrm{kg}$ pentobarbital sodium according to Rittirsch D's research[17]. A median incision (2 cm in length) was made on lower quadrants of abdominal wall, and the mesentery and cecum were 
dissociated under aseptic conditions. The cecum was exteriorized and ligated distal to the ileocecal valve about $3 \mathrm{~cm}$ with 4-0 non-absorbable suture. The cecum was perforated twice using a 22-gauge needle and a small amount of feces extruded through the perforation site. Animals were injected with saline solution $(5 \mathrm{ml} / 100 \mathrm{~g}$ ) subcutaneously (s.c.) immediately after the CLP surgery for fluid supplement. The rats were allowed free access to food and water after surgery. In the sham group, animals underwent all surgical procedures, but the cecum was neither ligated nor perforated. After the sepsis model successfully established, some rats were attacked by acute respiratory failure or renal failure. The survival rate was recorded every $24 \mathrm{~h}$ for seven days and the survival curve was plotted.

\section{Open field test}

Motor function and anxiety were assessed by the open field test (OFT). The open field apparatus was a black square $(100 \times 100 \mathrm{~cm}$; height of $50 \mathrm{~cm})$, and the arena was divided into the center area $(60 \mathrm{~cm} \times 60$ $\mathrm{cm}$ square) and the edge area (20 cm away from the walls). The light was maintained at minimum ( $300 \mathrm{~lx}$ ) to avoid anxiety behavior. The test sessions were recorded by a video camera placed $160 \mathrm{~cm}$ above the arena and analyzed with ANY maze Video Tracking System (Stoelting Co. Wood Dale, IL, USA). The experiment was performed on the third day after surgery. The total distance the animals travelled and duration in central area were measured in a ten minutes session. After one round of test, the arena was cleaned by $75 \%$ alcohol for eliminating odors.

\section{Morris water maze test}

Cognitive functions of spatial learning and memory were evaluated by Morris water maze test according to the methods of Wang[18]. The apparatus for the MWM consisted of a circular pool (diameter $180 \mathrm{~cm}$; height $45 \mathrm{~cm}$ ) filled with water made opaque with black dye $\left(22-24^{\circ} \mathrm{C}\right.$ ) and a platform (diameter $4 \mathrm{~cm}$, smooth-faced) hidden submerged $1 \mathrm{~cm}$ below the surface of the water located at a fixed location in the target quadrant. The maze was geographically divided into four quadrants that were equally spaced around the perimeter of the pool. The tests were recorded by a camera which was hanging above the pool and the results were analyzed by ANY maze Video Tracking System. The experiment, including the navigation and probe tests, was trained on the 3rd day after operation. Before the first day of MWM test, each rat was allowed to swim freely in water for one minute without the platform, in order to adapt the environment and eliminate unqualified rates. The navigation tests were performed on the third to sixth days post-CLP. During the continuous four training days, each rat was released into the pool to find a platform placed under the dark water $2 \mathrm{~cm}$ below, and trained four times from every different quadrant each day. On the fifth day of Morris water maze test, the platform was removed for space exploration training. The rats faced to the wall was placed in the water from the opposite side of the original platform quadrant. The time spent in the target quadrant and the times of crossing the platform were recorded.

\section{Resting-State MRI scan and fMRI data processing}

MRI scans were performed in a 7 Tesla horizontal bore magnet (Bruker Biospec 7 T/20 USR; Bruker, Karlsruhe, Germany). Using a rat head volume coil, we acquired blood oxygen level dependent (BOLD) 
fMRI responses. Eight rats in each group were included in imaging examinations. Fast spin-echo T2 weighted MR images were obtained to visualize the structure of the rats' brains. The rats were anesthetized by $2 \%$ isoflurane, and then, the head was fixed in a capsule with a tooth-bar to limit the movement. Inhaling with $2 \%$ isoflurane and $2 \mathrm{~L} / \mathrm{min}$ oxygen, the rats breathed at a rate of 60 times per minute. Fast spin-echo T2 weighted MR images were obtained with the following parameters: slice $=25$, repetition time $(T R)=3000 \mathrm{~ms}$, echo time $(T E)=11 \mathrm{~ms}, T A=4 \min 48 \mathrm{~s}, \mathrm{SI}=1.00 / 1.00 \mathrm{~mm}, \mathrm{FOV}=3.17 / 2.50$ $\mathrm{cm}, \mathrm{MTX}=256$. The scan parameters of BOLD: slice $=18, T R=2000 \mathrm{~ms}, T E=25 \mathrm{~ms}$, Slice Thickness $=1 \mathrm{~mm}$, $\mathrm{TA}=5 \mathrm{~min}$, image size $=128 \times 128$.

After preprocessing of EPI series (discarding the first 10 volumes, slice timing, correction, realignment, motion correction, coregistration with T2 images to rat brain atlas) with SPM8 (https://www.fil.ion.ucl.ac.uk/spm/) and DPABI (http: //rfmri.org/dpabi) [19], the normalized images were linearly detrended. Those on which the head moved by more than $0.2 \mathrm{~mm}$ and 2 degree were excluded. After the images were smoothed with a Gaussian kernel of $4 \mathrm{~mm} \times 4 \mathrm{~mm} \times 4 \mathrm{~mm}$ full width at halfmaximum (FWHM) to improve the signal-to-noise ratio (SNR), amplitude of the low-frequency fluctuations (ALFF) was abstracted from the BOLD images. For rs-fMRI data, regions of interest (ROIs) were extracted from SIGMA Anatomical Brain Atlas using dpabi software, four brain regions were chosen as seed ROIs for analyses of brain functional connection. The functional connectivity (FC) score between CA1 of hippocampus and lateral entorhinal cortex (LEnt) on the same side of the cerebral hemisphere were calculated through using Pearson's correlation coefficients and converted to normally distributed Zscores using the Fisher transformation.

\section{Enzyme-linked immunosorbent assay}

Blood was collected from left ventricle, and then samples were centrifuged at $12000 \mathrm{~g}$ for $10 \mathrm{~min}$ at $4{ }^{\circ} \mathrm{C}$. The serum levels of IL-1 $\beta$, IL-6, CRP were determined by ELISA kits (Solarbio, Beijing, China) according to the manufacturer's instructions, and the absorbance was measured with a micro-plate reader at $450 \mathrm{~nm}$ (optical density (OD) value).

\section{Western blot analysis}

The hippocampus on both sides were lysed and homogenized in RIPA lysis buffer containing phenylmethylsulfonyl fluoride immediately. After protein concentration assayed with the BCA kit, Total protein samples were separated by $12 \%$ sodium dodecyl sulfate polyacrylamide gel electrophoresis (SDS-PAGE) and transferred to polyvinylidene difluoride (PVDF) filters. Membranes were probed with antirat monoclonal antibodies raised in rats against MFG-E8 (Abcam, ab9324), Rac1 (Abcam, ab155938) and LC3 (Abcam, 192890), overnight at $4^{\circ} \mathrm{C}$. On the second day, the membrane were washed three times using PBS with $0.1 \%$ Tween 20 , reacted with HRP-conjugated affinipure rabbit Anti-rat IgG $(\mathrm{H}+\mathrm{L})$ (Abcam, ab6734) for two hours at $22-25^{\circ} \mathrm{C}$. The integral optical density (IOD) of each band was measured using ImageJ software.

\section{Hematoxylin-Eosin (HE) Staining for pathology observation}


After 48 hours of fixation with $4 \%$ paraformaldehyde, brain tissues were cut into slices in the coronal direction. Brain slices were dehydrated with gradient alcohol, cleared in xylene, and embedded in paraffin.

Subsequently, brain tissues were cut into $5-\mu \mathrm{m}$ slides for HE staining (Boster, Wuhan, China) and captured under a microscope.

\section{Statistical Analysis}

Data presented as mean \pm standard deviation (S.D.) was analyzed with SPSS 22.0 and graph pad prism 8. Multiple groups comparisons were performed by one-way ANOVA followed by Tukey test for multiple comparisons. In behavior test results, western blot results and ELISA results, $\mathrm{p}$-value less than $0.05(p<$ 0.05 ) was considered statistically significant. The difference of ALFF value was analysed by two sample t-tests with REST software $(p<0.001)$. The ALFF value were compared between CLP group and CLP +MFGE8 group, as well as between CLP group and CLP +MFGE8+cilengitide group. The correction thresholds were limited by false discovery rate (FDR) $p=0.05$, cluster connectivity criterion: $\mathrm{mm}=5$, cluster size $=21, p=0.001$.

The zFC between CA1 and LEnt on the same side of the cerebral hemisphere were analysed by one-way ANOVA followed by Tukey test. The relationships between FC of hippocampal CA1 and LEnt and total time in target quadrant in Morris water maze test were explored by Spearman's correlation analysis.

\section{Results}

\section{MFGE8 improved post-operation 7-day survival rate in SAE rats}

Cecal ligation and puncture surgery induced sepsis rats died for serious systemic inflammatory response and multiple organ failure. The post-operation 7-day survival rate indicated the severity of sepsis in different groups. The post operation 7-day survival rate of rats respectively in four groups were $100 \%$ in sham group, $63.03 \%$ in CLP group, 50.79\% in CLP + MFGE8 group, and 38.96\% in CLP + MFGE8 + Cilengitide group (Fig. 1a).

\section{MFGE8 improved performance of neurobehavioral tests}

To test the motor function and anxiety of rats in four groups, we recorded the total running distance and duration in central area by the open field test (OFT)(Fig. 1b). It was shown that there was no significantly difference in the total running distance among four groups (Fig. 1c; $p>0.05$ ). The duration in central area in CLP group was less than that in sham group. MFGE8 improved exploration behavior of rats, while MFGE8 antagonist cilengitide reversed the effects (Fig. $1 \mathrm{~d} ; p<0.01$ ).

To evaluate the cognitive dysfunction among rats in four groups, we test the rats by Morris water maze task (Fig. 1f). It was shown that MFGE8 shorten the studying time in the training section (Fig. 1e; $p<$ 0.01), and improved the time spent in the target quadrant and the times of crossing the platform in space exploration section (Fig. 1g; $p<0.001$ ). While MFGE8 antagonist cilengitide caused significantly impaired spatial memory (Fig. 1h; $p<0.001$ ). 


\section{MFGE8 changed the expression of inflammatory factor in serum by ELISA}

We examined the expression of interleukin $1 \beta$ (IL-1ß) (Fig. 2a), interleukin 6 (IL-6) (Fig. 2b), and C-reactive protein (CRP) (Fig. 2c) in serum. It was observed that IL-1 3 , IL-6 and CRP levels in CLP group are notably higher than those in sham group $(p<0.01)$. And in CLP+MFGE8 group, the expression of IL-1 $\beta$ increased than CLP group $(p<0.01)$. However, the expression of IL-6 decreased in CLP+MFGE8+cilengitide group compared with that in CLP group $(p<0.01)$. The difference of CRP among three CLP group were not obviously.

\section{Expression of efferocytosis related proteins up-regulated in CLP+MFGE8 group}

MFGE8 could reflect the binding ability of microglia to apoptotic fragments. The expression of efferocytosis related proteins in bilateral hippocampus were examined after surgery eight days for four groups(Fig. 2e). The expression of MFGE8 in CLP+MFGE8 group tended to increase, while the expression of MFG-E8 decreased in CLP+MFGE8+cilengitide group. (Fig. 2f; $p<0.01$ ). Injection of MFGE8 increased the expression of Rac1 in hippocampus after CLP surgery, while addition with cilengitide, the aV $\beta 3$ and aV $\beta 5$ integrin inhibitor, down regulated the expression of Rac1 which played a vital role in migration of microglia. (Fig. 2d; $p<0.01$ ). LC3 is a key molecule in intracellular phagocytosis during efferocytosis, which can reflect the digestive function of microglia. Cecal ligation and puncture surgery induced sepsis model had severe destroyed digestive function, and apoptotic cell fragments were accumulated in hippocampus. Microglial phagocytosis deteriorated sharply after the inhibitor of integrin receptor cilengitide injected in CLP+ MFGE8+cile group (Fig. 2g; $p<0.01$ ).

\section{MFGE8 reduced hyperintense volume in the hippocampus, and increased ALFF value in SAE rats' cortex}

T2-MRI can detect the structure changes in brain of rats after CLP surgery. Compared with sham group, the signals in alveus of the hippocampus (alv) and external capsule (ec) were increased in CLP group. The hyperintense focus in the region of hippocampus, indicating the brain cells were edema seven days after sepsis. In CLP+MFGE8 group, the hyperintense region were significantly decreased in hippocampus. Abnormally elevated signals of cerebrospinal fluid are clustered in the amygdalopiriform transition area (APir), suggesting the supplement of MFGE8 could reduce the brain damage among SAE rats. Cilengitide inhibited the therapeutic function of MFGE8, increased the high signal regions, show more serious damages in the hippocampus (Fig. 3).

Sepsis rats with encephalopathy underwent MRI scan, showing that a regional signal increase was produced in the hippocampus. However, this abnormal signals were reduced in CLP+MFGE8 group. In CLP+MFGE8+cilengitide group, the hyperintense focus in the region of hippocampus, and the range of damage areas were significantly large than that in CLP group.

MFGE8 increased ALFF value in SAE rats' cortex. The rs-fMRI technology was employed to examine the changes in brain function in SAE rats. Notably, the ALFF value of the bilateral sensory cortex, motor cortex and visual cortex were higher in SAE+MFGE8 group rats relative to SAE rats in CLP group.(Fig. 4a; 
red areas). To the contrary, cilengitide deteriorated the brain function especially in the auditory cortex, lateral entorhinal cortex (LEnt), perirhinal cortex (PRh), and dorsal hippocampal commissure (Dhc). (Fig. $4 \mathrm{~b}$; blue areas). Particularly, the functional deterioration occurred on both sides of cerebral hemisphere.

\section{MFGE8 increased FC of CA1 and LEnt in same side of cerebral hemisphere, and the relationship between FC of CA1 and LEnt and behavior test result of total time in target quadrant were significant positive correlations.}

As we found that cilengitide deteriorated the brain function in bilateral lateral entorhinal cortex (LEnt), and these brain areas had close relationship with hippocampus in neuron fibers connection. We analyzed the difference of functional connectivity between CA1 of hippocampus and lateral entorhinal cortex, and evaluated the correlation relationship between $\mathrm{FC}$ and behavior test results. The four regions were extracted from SIGMA Anatomical Brain Atlas, left CA1 of hippocampus (pink area), right CA1 of hippocampus (green area), left LEnt (red area) and right LEnt (blue area) were defined as ROIs. (Fig. 5a). The results showed that FC between the left CA1 and LEnt were decreased in CLP group and CLP+MFGE8+cilengitide group compared with CLP+MFGE8 group. (Fig. $5 \mathrm{~b} ; p<0.01, N=8$ ). And the FC were positively correlated with total time in target quadrant in MWM test which reflected the capacity of episodic memory (Fig. 5c; $r^{2}=0.3183, p=0.0008$ ). Meanwhile, the same trend was observed in the right hemisphere, the $\mathrm{FC}$ between the right CA1 and LEnt were also reduced in CLP group and CLP+MFGE8+cilengitide group. (Fig. $5 \mathrm{~d} ; p<0.01, N=8$ ). The relationship between FC and behavior result were also a positive correlation (Fig. $5 e ; r^{2}=0.3429, p=0.0004$ ).

\section{The effects of MFGE8 and cilengitide on brain pathological changes with H\&E staining}

The morphology of neurons in the hippocampus was normal in the sham group, which showed a normal hippocampal structure and the pyramidal cells are arranged orderly. In CLP group, some microglial cells approached to the pyramidal cells, and more gliocyte cells congregate in granular cell layer. Vascular walls in brain became thicken, and the cellular swelling impede the process of microglial cells' migration. In CLP+MFGE8 group, activated microglia which was in a rod-shaped nucleus, accumulated in the white mater. The number of cells in granulosa cell layer was significantly less than that of SAE group. In CLP+MFGE8+cilengitide group, more severe damage to neuron was observed in the hippocampus. The neuronal cells were lysis, and the staining in cytoplasm was enhanced homogeneously. (Fig. 6).

\section{Discussion}

Our study shows that MFGE8 promoted efferocytosis with microglia and improved brain function which were detected by behavior tests, as well as repaired brain structure and function connection detected by fMRI and pathological staining.

Neuroinflammation and accumulation of apoptotic neuron are thought to play a sustaining pathogenic role in sepsis associated encephalopathy. Specially, microglial cell appears to be a key factor both in process of generation of inflammation factor and elimination of apoptotic cells[20]. Accordingly, 
regulating activation of microglia cells may serve a promising therapeutic approach in early management of SAE[21]. Presently, it is controversial that repressing activation of microglia cells alleviated inflammation in SAE rats [22, 23]. Previous studies have shown that MFGE8 expression decreased in experimental sepsis, and the supplement of MFG-E8 treatment had therapeutic potential for sepsis [6, 24]. MFGE8 was secreted by microglia and astrocytes in the central nervous system, and attached to the aV $\beta 3$ and $\mathrm{aV} \beta 5$ integrins on the microglial surface, for recognizing and enclosing the apoptotic cells[12]. Several researches had confirmed that the anti-apoptotic effect of MFGE8 in central never system disease, for instances in the alcoholic encephalopathy, as well as in experimental models of subarachnoid hemorrhage (SAH). However, the therapeutic effect of MFGE8 activating microglia in SAE has not been confirmed.

In the present study, we first confirmed the supplement of exogenous MFGE8 alleviated the neuronal death in hippocampus of SAE rats. After intracerebroventricular injection with MFGE8 3.3ug, the activated microglia gathered in hippocampus, and pathological lesion mitigated. The elevation of MFGE8 occurred obviously in CLP+MFGE8 group, in parallel with the change of downstream molecules in efferocytosis such as Rac 1 and LC3. Meanwhile, the level of inflammatory factors in plasma, such as IL-6, CRP, and IL$1 \beta$ were decreased in CLP+MFGE8 group. We also confirmed that MFGE8 could significantly decrease the abnormal area of high signal in hippocampus by T2-MRI scan. To the contrary, with the intervention of cilengitide, inhibitor of aV $\beta 3$ and $\mathrm{aV} \beta 5$ integrins on the microglial surface, apoptosis cells were accumulated, efferocytosis related proteins were less than those in CLP+MFGE8 group, and the inflammatory factors were also down regulated. Cilengitide, a selective av $\beta 3$ and av $\beta 5$ integrin inhibitor, had been used in clinical treating glioblastoma [25]. However, the clinical application did not improve outcomes for patients with glioblastoma[26]. From the results of WB (Fig2. d-g), cilengitide inhibited the microglial phagocytosis which was actived by av $\beta 3$ and av $\beta 5$ integrin inhibitor. Furthermore, LC3 is a key protein in autophagy, which was also expressed in normal neurons[27]. Rac1 is the downstream protein of $\operatorname{av} \beta 3$ and av $\beta 5$ integrin receptor, which regulated the processes such as cell migration, invasion and DNA damage[28]. Meanwhile, the trend of Rac1 and LC3 could reflect the phagocytosis of microglia in the hippocampus. In our research, we found that efferocytosis of microglia was inhibited in the sepsis models induced by CLP surgery. Furthermore, under the premise of MFGE8 intervention, while using cilengitide intervention, migration and phagocytosis of Microglia were inhibited, which was showed expressions of Rac1 and LC3 decreased. Taken together, these results confirmed that MFGE8 is a key molecular in the efferocytosis during the process of microglia eliminating apoptotic cells.

In addition, a major strength of our study involves the 7 Tesla structural MRI scan we conducted, allowing us to identify structural changes in areas as small as the amygdalopiriform transition area (APir). In CLP group, the signals in alveus of the hippocampus (alv) and external capsule (ec) were increased than those in sham group. However, the lesion was only confined to the cerebrospinal fluid, while the hippocampus which adjoined to abnormal areas kept relatively normal structure. The voxel of corpus callosum (cc) and cingulate cortex (cg) increased in CLP group, indicating brain cellular edema happened in rats with sepsis associated encephalopathy. Furthermore, we compared the effect of MFGE8 with receptor antagonist cilengitide in SAE rats. In this study, abnormally elevated signals of cerebrospinal 
fluid are clustered in the areas amygdalopiriform transition area (APir) and lateral ventricle (LV). But the diseased areas alleviated significantly than CLP group with T2 MRI. In comparison with CLP group, the gray matter in hippocampus injured more serious in CLP+MFGE8+cilengitide group. Almost total right hippocampus damaged, while the brain middle basal was pushed to the left. Studies had confirmed that aV 33 and $\mathrm{aV} \beta 5$ integrins receptor were not only expressed on activated microglial cells, but also on activated endothelial cells[29]. Furthermore, the $\mathrm{aV} \beta 3$ and $\mathrm{aV} \beta 5$ receptor antagonist cilengitide caused blood brain barrier (BBB) breakdown by injuring the junction of endothelial cells. Large amounts of toxins entered the brain parenchyma, meanwhile the phagocytosis of microglial cells was inhibited, and the results were serious damages occurred in sepsis rats with cilengitide injection. Combined with the results of serum inflammatory factors, we found that the IL- $1 \beta$ and IL- 6 were down regulated after supplemented with cilengitide, indicating that the activation of microglial cells was repressed. However, in terms of the behavioristics results, it didn't get worse with cilengitide injected. And the damaged areas of gray matter did not have an obvious correlation with results of MWM and OFT.

In the subsequent study, we also assessed cognitive function using rs-fMRI technology by observing the ALFF value change. The amplitude of low-frequency fluctuation (ALFF) is one of the analysis strategies on functional data, which was based on blood oxygenation level-dependent (BOLD) signals[30]. ALFF reflects the intensity of spontaneous fluctuations in local regions, which reveals the average spontaneous neural activity during scanning[31]. Increase in ALFF indicates enhanced brain activity. In contrast, decrease in ALFF indicates that the spontaneous activity of neurons in the corresponding brain area is weakened. In this study, the ALFF value of the bilateral sensory cortex, motor cortex and visual cortex increased in SAE+MFGE8 group, indicating the brain function activated in brain cortex. With cilengitide, the brain function was inhibited in auditory cortex, lateral entorhinal cortex (LEnt), perirhinal cortex (PRh), and dorsal hippocampal commissure (Dhc). These changes illustrated that MFGE8 improved brain function, and this protein played important role in normal cognition function.

We also evaluated the functional connectivity between CA1 of hippocampus and lateral entorhinal cortex (LEnt), which were confirmed necessary in spatial memory. Some researches had confirmed that the functional connectivity of the hippocampus with its primary cortical input, the entorhinal cortex, is organized topographically[32]. In this study, we found the functional connectivity of CA1 and LEnt was different in four groups, and the FC values were low in those groups which were performed worse in Morris water maze test. Yiding's research showed that lateral entorhinal cortex (LEC)-dCA1 pyramidal cells direct circuit that is critically involved in hippocampal-dependent olfactory associative learning[33]. We note that the FC value were positive correlated with total time in target quadrant in MWM test. This is consistent with previous findings that LEC-CA1 direct circuit controlled hippocampal related learning behavior.

There may be some possible limitations in this study. As the learning behavior and memory involved many brain regions, we only evaluated the LEnt-CA1 functional connectivity in this study. We need to select other brain regions with different functional activation, such as medial prefrontal cortex (mPFC), amygdala, and marginaldivision of the striatum (MrD) to evaluate the brain function of SAE rats. The 
second limitation concerns the analytical method of fMRI results. More advanced analytical methods will be needed to understand how brain function in SAE rats various in learning and memory.

\section{Conclusion}

In conclusion, our study has elucidated the function of MFGE8 on efferocytosis in sepsis associated encephalopathy, and discussed the value of $\mathrm{fMRI}$ in the study of sepsis related cognitive impairment in rats. We highlight the role of MFGE8 in promoting efferocytosis by activating aV $\beta 3$ and aV $\beta 5$ receptor on microglia surface and improving brain function which were detected by behavior tests and fMRI. This study also elucidated the functional connectivity of CA1 and LEnt was consistent with the behavior result in Morris water maze test.

\section{Abbreviations}

AC: apoptotic cell; ALFF: amplitude of the low-frequency fluctuations; BBB: blood brain barrier; BOLD: Blood oxygenation level dependent; CA1: Cornu Ammonis-1; CLP: cecal ligation and puncture; CNS: central nervous system; CT: computer tomography; Dhc: dorsal hippocampal commissure; EGF: epidermal growth factor; ELISA: Enzyme-linked immunosorbent assay; FC: Functional connectivity; FWHM: full width at half-maximum; LC3: Microtubule-associated protein light chain 3; LEnt: lateral entorhinal cortex; MFG-E8: Milk fat globule EGF factor 8 protein; MRI: magnetic resonance imaging; MWM: Morris water maze; OFT:open filed test; PRh: perirhinal cortex; PS: phosphoryl serine; Rac1: rasrelated C3 botulinum toxin substrate 1; ROI: region of interest; rs-fMRI: Resting state functional magnetic resonance imaging; SAE: Sepsis-associated encephalopathy; TE: echo time; TR: repetition time

\section{Declarations}

\section{Acknowledgments}

We thank Dandan Liang for her help with the MRI scan; Xue Liu, Hayan Zhu, Jingjing Song, and Lingyuan Zhang for their help with western blot and behavior test; Wenbin Zhou, Yali Zhai and Yuxing Hou for their help with pathological analysis; Jing Xiao for his critical comments on the manuscript.

\section{Ethics approval and consent to participate}

All animal procedures were approved by the Institutional Animal Care and Use Committee of Nanjing Medical University(IACUC: 2103051).

\section{Consent for publication}

Not applicable

\section{Competing interests}


The authors declare that they have no competing interests.

\section{Availability of data and materials}

The data that support the findings of this study are available from corresponding authors upon reasonable request.

\section{Funding}

This work was supported by grants from the National Natural Science Foundation of China (grant numbers: 81971872₫81873954).

\section{Authors' contributions}

$H J L, H S L$, and HB designed the research;

HJL, HSL, ZZ, and JD performed the research;

HJL, YS, YC, and $X X$ collected and analyzed the data;

YS and HB provided administrative support;

HJL, HSL, and HB drafted the manuscript.

All authors edited and approve the manuscript.

\section{References}

1. Rudd KE, Johnson SC, Agesa KM, Shackelford KA, Tsoi D, Kievlan DR, Colombara DV, Ikuta KS, Kissoon N, Finfer S et al. Global, regional, and national sepsis incidence and mortality, 1990-2017: analysis for the Global Burden of Disease Study. The Lancet. 2020,395(10219):200211.doi:10.1016/s0140-6736(19)32989-7

2. Chung HY, Wickel J, Brunkhorst FM, Geis C. Sepsis-Associated Encephalopathy: From Delirium to Dementia? J Clin Med. 2020,9(3).doi:10.3390/jcm9030703

3. Kawabe K, Takano K, Moriyama M, Nakamura Y. Microglia Endocytose Amyloid beta Through the Binding of Transglutaminase 2 and Milk Fat Globule EGF Factor 8 Protein. Neurochem Res. 2018,43(1):41-49.doi:10.1007/s11064-017-2284-y

4. Chen K, Murao A, Arif A, Takizawa S, Jin H, Jiang J, Aziz M, Wang P. Inhibition of Efferocytosis by Extracellular CIRP-Induced Neutrophil Extracellular Traps. The Journal of Immunology. 2021,206:797-806.doi: 10.1016/j.biomaterials.2021.121163.

5. Zhang L, Tian R, Yao X, Zhang XJ, Zhang P, Huang Y, She ZG, Li H, Ji YX, Cai J. Milk Fat GlobuleEpidermal Growth Factor-Factor 8 Improves Hepatic Steatosis and Inflammation. Hepatology. 2021,73(2):586-605.doi:10.1002/hep.31277 
6. Chaung WW, Brenner M, Yen HT, Ochani ML, Jacob A, Wang P. Recombinant human milk fat globuleEGF factor VIII (rhMFG-E8) as a therapy for sepsis after acute exposure to alcohol. Mol Med. 2019,25(1):52.doi:10.1186/s10020-019-0118-x

7. Tauber SC, Djukic M, Gossner J, Eiffert H, Bruck W, Nau R. Sepsis-associated encephalopathy and septic encephalitis: an update. Expert Rev Anti Infect Ther. 2021,19(2):215231.doi:10.1080/14787210.2020.1812384

8. Ding H, Li Y, Chen S, Wen Y, Zhang S, Luo E, Li X, Zhong W, Zeng H. Fisetin ameliorates cognitive impairment by activating mitophagy and suppressing neuroinflammation in rats with sepsisassociated encephalopathy. CNS Neuroscience \& Therapeutics. 2022,28(2):247258. .doi:10.1111/cns.13765

9. Li G, Han X, Gao W, Song Z, Zhao S, Sun F, Ma H, Cui A, Tang X, Ma G. Influence of EGR3 Transfection on Imaging and Behavior in Rats and Therapeutic Effect of Risperidone in Schizophrenia Model. Front Psychiatry. 2020,11:00787.doi:10.3389/fpsyt.2020.00787

10. Becq GJC, Barbier EL, Achard S. Brain networks of rats under anesthesia using resting-state fMRI: comparison with dead rats, random noise and generative models of networks. J Neural Eng. 2020,17(4):045012.doi:10.1088/1741-2552/ab9fec

11. Zhu X, Ji MH, Li SM, Li B, Mei L, Yang JJ. Systemic Inflammation Impairs Mood Function by Disrupting the Resting-State Functional Network in a Rat Animal Model Induced by Lipopolysaccharide Challenge. Mediators Inflamm. 2019,2019:6212934.doi:10.1155/2019/6212934

12. Luo X, Ren Q, Luo M, Li T, Lv Y, Liu Y, Rong K, Zhang W, Li X. Glutamate Chemical Exchange Saturation Transfer Imaging and Functional Alterations of Hippocampus in Rat Depression Model: A Pilot Study. J Magn Reson Imaging. 2021,54(6):1967-1976.doi:10.1002/jmri.27850

13. Gao R, Ji MH, Gao DP, Yang RH, Zhang SG, Yang JJ, Shen JC. Neuroinflammation-Induced Downregulation of Hippocampacal Neuregulin 1-ErbB4 Signaling in the Parvalbumin Interneurons Might Contribute to Cognitive Impairment in a Mouse Model of Sepsis-Associated Encephalopathy. Inflammation. 2017,40(2):387-400.doi:10.1007/s10753-016-0484-2

14. Liu F, Chen Y, Hu Q, Li B, Tang J, He Y, Guo Z, Feng H, Tang J, Zhang JH. MFGE8/Integrin beta3 pathway alleviates apoptosis and inflammation in early brain injury after subarachnoid hemorrhage in rats. Exp Neurol. 2015,272:120-127.doi:10.1016/j.expneurol.2015.04.016

15. Desgrosellier JS, Cheresh DA. Integrins in cancer: biological implications and therapeutic opportunities. Nat Rev Cancer. 2010,10(1):9-22.doi:10.1038/nrc2748

16. Wang J, Wang Y, Zuo Y, Duan J, Pan A, Li JM, Yan XX, Liu F. MFGE8 mitigates brain injury in a rat model of SAH by maintaining vascular endothelial integrity via TIGbeta5/PI3K/CXCL12 signaling. Exp Brain Res. 2021,239(7):2193-2205.doi:10.1007/s00221-021-06111-x

17. Rittirsch D, Huber-Lang MS, Flierl MA, Ward PA. Immunodesign of experimental sepsis by cecal ligation and puncture. Nat Protoc. 2009,4(1):31-36.doi:10.1038/nprot.2008.214

18. Wang W, Liu L, Jiang P, Chen C, Zhang T. Levodopa improves learning and memory ability on global cerebral ischemia-reperfusion injured rats in the Morris water maze test. Neurosci Lett. 2017,636:233- 
240.doi:10.1016/j.neulet.2016.11.026

19. Yan CG, Wang XD, Zuo XN, Zang YF. DPABI: Data Processing \& Analysis for (Resting-State) Brain Imaging. Neuroinformatics. 2016,14(3):339-351.doi:10.1007/s12021-016-9299-4

20. Sui DM, Xie Q, Yi WJ, Gupta S, Yu XY, Li JB, Wang J, Wang JF, Deng XM. Resveratrol Protects against Sepsis-Associated Encephalopathy and Inhibits the NLRP3/IL-1beta Axis in Microglia. Mediators Inflamm. 2016,2016:1045657.doi:10.1155/2016/1045657

21. Ye B, Tao T, Zhao A, Wen L, He X, Liu Y, Fu Q, Mi W, Lou J. Blockade of IL-17A/IL-17R Pathway Protected Mice from Sepsis-Associated Encephalopathy by Inhibition of Microglia Activation. Mediators Inflamm. 2019,2019:8461725.doi:10.1155/2019/8461725

22. Xie K, Zhang Y, Wang Y, Meng X, Wang Y, Yu Y, Chen H. Hydrogen attenuates sepsis-associated encephalopathy by NRF2 mediated NLRP3 pathway inactivation. Inflamm Res. 2020,69(7):697710.doi:10.1007/s00011-020-01347-9

23. Li Y, Yin L, Fan Z, Su B, Chen Y, Ma Y, Zhong Y, Hou W, Fang Z, Zhang X. Microglia: A Potential Therapeutic Target for Sepsis-Associated Encephalopathy and Sepsis-Associated Chronic Pain. Front Pharmacol. 2020,11:600421.doi:10.3389/fphar.2020.600421

24. Matsuda A, Jacob A, Wu R, Zhou M, Nicastro JM, Coppa GF, Wang P. Milk fat globule-EGF factor VIII in sepsis and ischemia-reperfusion injury. Mol Med. 2011,17(1-2):126133.doi:10.2119/molmed.2010.00135

25. Reardon DA, Nabors LB, Stupp R, Mikkelsen T. Cilengitide: an integrin-targeting arginine-glycineaspartic acid peptide with promising activity for glioblastoma multiforme. Expert Opin Investig Drugs. 2008,17(8):1225-1235.doi:10.1517/13543784.17.8.1225

26. Stupp R, Hegi ME, Gorlia T, Erridge SC, Perry J, Hong Y-K, Aldape KD, Lhermitte B, Pietsch T, Grujicic D et al. Cilengitide combined with standard treatment for patients with newly diagnosed glioblastoma with methylated MGMT promoter (CENTRIC EORTC 26071-22072 study): a multicentre, randomised, open-label, phase 3 trial. The Lancet Oncology. 2014,15(10):1100-1108.doi:10.1016/s14702045(14)70379-1

27. Kim EY, Dryer SE. RAGE and alphaVbeta3-integrin are essential for suPAR signaling in podocytes. Biochim Biophys Acta Mol Basis Dis. 2021,1867(10):166186.doi:10.1016/j.bbadis.2021.166186

28. Payapilly A, Malliri A. Compartmentalisation of RAC1 signalling. Curr Opin Cell Biol. 2018,54:5056.doi:10.1016/j.ceb.2018.04.009

29. Keasey MP, Jia C, Pimentel LF, Sante RR, Lovins C, Hagg T. Blood vitronectin is a major activator of LIF and IL-6 in the brain through integrin-FAK and uPAR signaling. J Cell Sci. 2018,131(3).doi:10.1242/jcs.202580

30. Haak KV, Marquand AF, Beckmann CF. Connectopic mapping with resting-state fMRI. Neuroimage. 2018,170:83-94.doi:10.1016/j.neuroimage.2017.06.075

31. Wang X, Wang Q, Zhang P, Qian S, Liu S, Liu DQ. Reducing Inter-Site Variability for Fluctuation Amplitude Metrics in Multisite Resting State BOLD-fMRI Data. Neuroinformatics. 2021,19(1):2338.doi:10.1007/s12021-020-09463-x 
32. Kumar M, Deshmukh SS. Differential propagation of ripples along the proximodistal and septotemporal axes of dorsal CA1 of rats. Hippocampus. 2020,30(9):970-

986.doi:10.1002/hipo.23211

33. Li Y, Xu J, Liu Y, Zhu J, Liu N, Zeng W, Huang N, Rasch MJ, Jiang H, Gu X et al. A distinct entorhinal cortex to hippocampal CA1 direct circuit for olfactory associative learning. Nat Neurosci. 2017,20(4):559-570.doi:10.1038/nn.4517

\section{Figures}

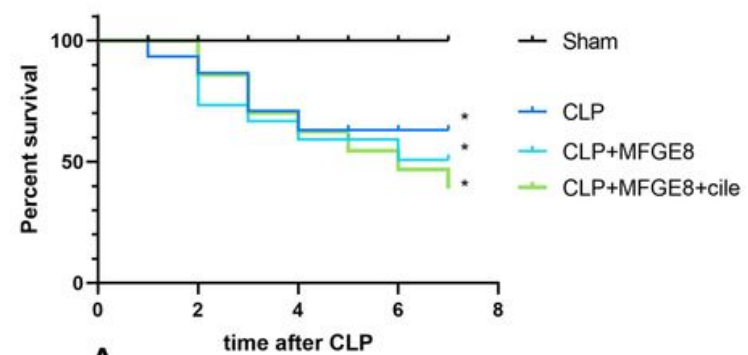

A

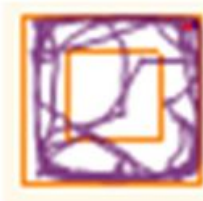

Sham

B

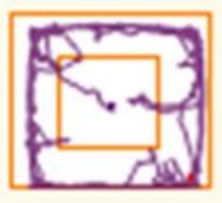

CLP

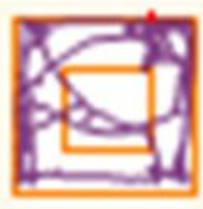

CLP+MFGE8

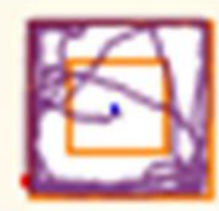

CLP+MFGE8+cilen
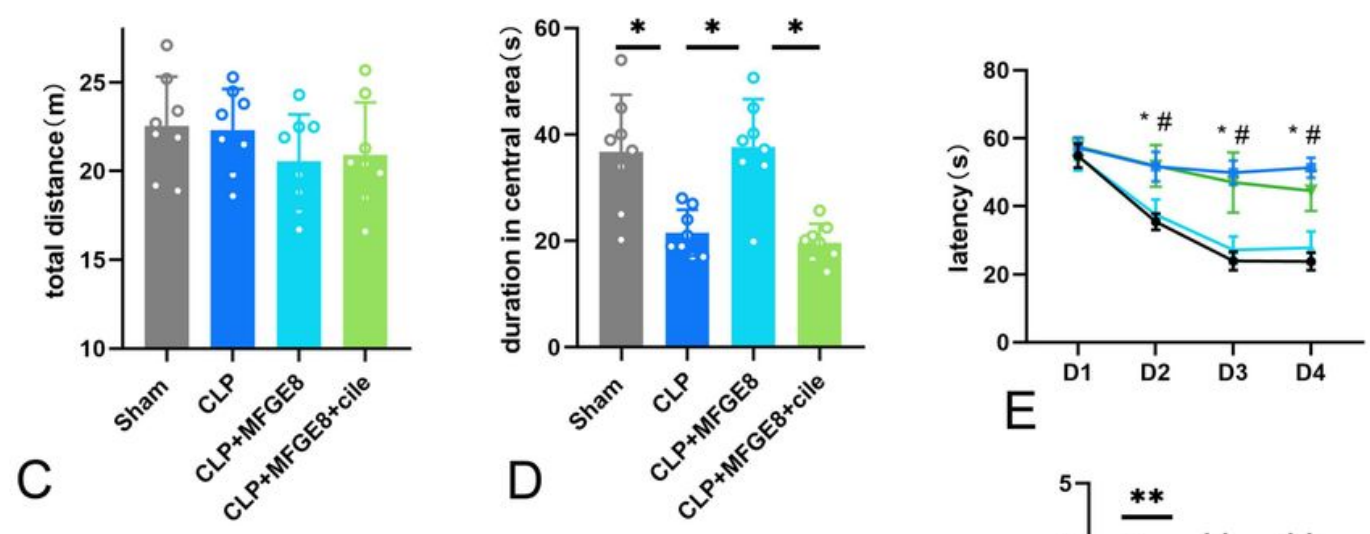

$\rightarrow$ Sham

- CLP

- CLP+MFGE8

* CLP+MFGE8+cile

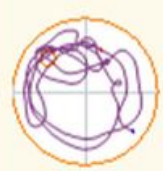

Sham

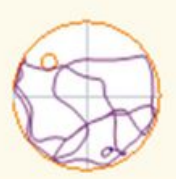

CLP

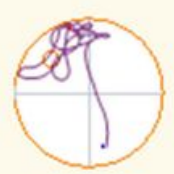

CLP+MFGE8

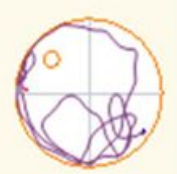

CLP+MFGE8+cilen

$\mathrm{F}$

\section{Figure 1}

Survival rates and neurobehavioral tests results 
(a) Survival rates after CLP surgery. Respectively, the seven-days survival proportion in sham group, CLP group, CLP+MFGE8 group and CLP+MFGE8+cilengitide group were $100 \%, 63.03 \%, 50.79 \%$ and $38.96 \%$.

(b) The tracks of rats in open field test. (c)The total distance the rats traveled in 10 minutes $(F(3,28)$ $=1.122, P>0.05$, one way ANOVA, $N=8)$. (d) Duration in central area the rats traveled in 10 minutes $(\mathrm{F}(3$, $28)=13.08, P<0.05$, ANOVA, $* p<0.01, N=8)$. (e) The difference of latency in navigation test in MWM during four days. ( ${ }^{*} p<0.01, N=8$, compared with sham group, $\# p<0.01, N=8$, compared with CLP+MFGE8 group). (f) The tracks of rats in probe tests of MWM. (g) The number of crossings of the platform location $\left(F(3,28)=14.42\right.$, ANOVA, $\left.{ }^{*} p<0.001, N=8\right)$. (h) The total time in target quadrant $(F(3$, 28) $=10.23,{ }^{\star \star} p<0.001$, repeated measurements ANOVA, $\left.N=8\right)$.
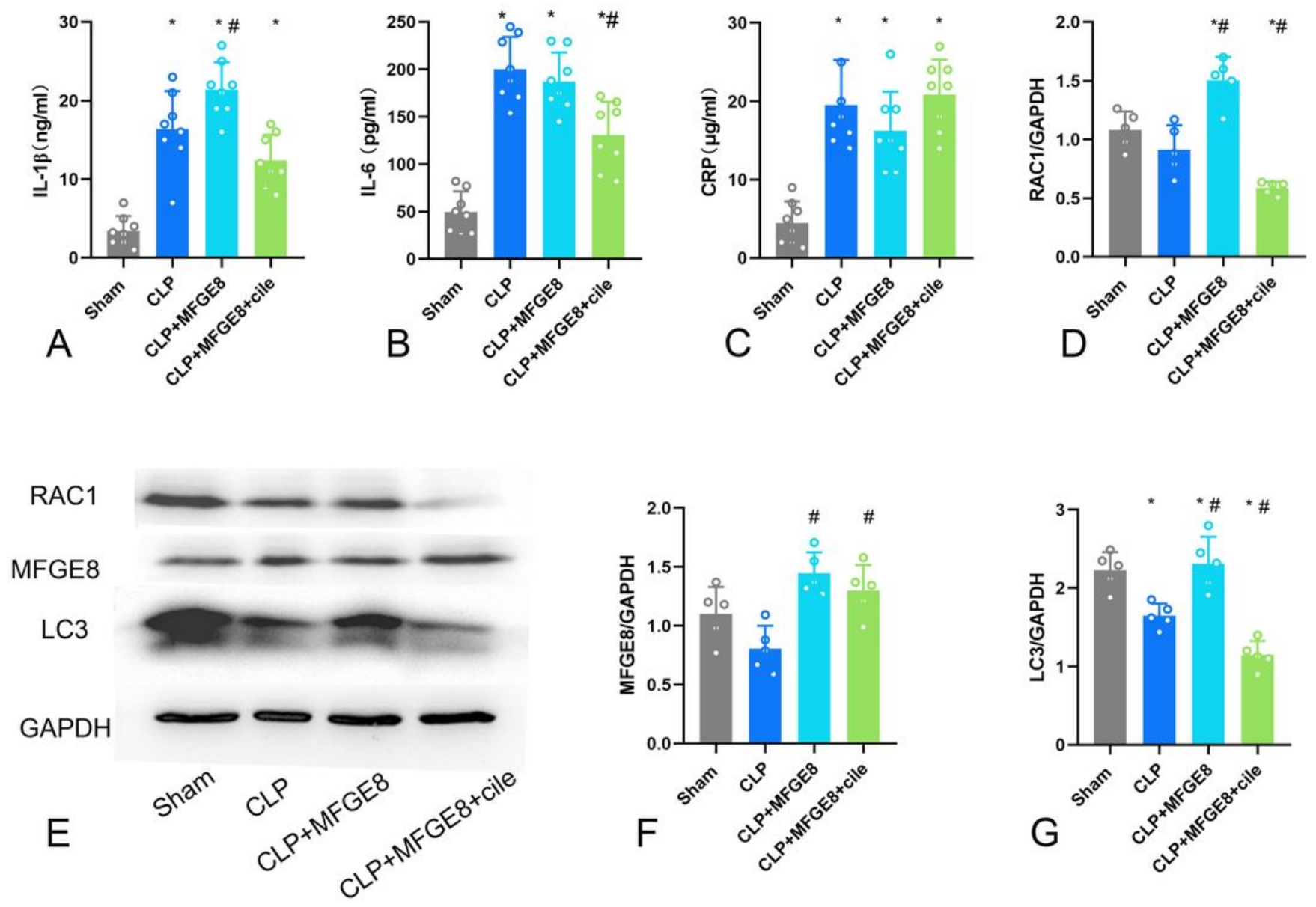

Figure 2

Inflammatory factor in serum by ELISA and efferocytosis related proteins in hippocampus by western blot

(a) Expression of IL-1 $\beta$, (b) interleukin 6 (IL-6), (c) C-reactive protein (CRP) in serum $\left({ }^{*} p<0.01\right.$, compared with sham group; $\# p<0.01$, compared with CLP group, one way ANOVA, $N=5$ ). (e) Expression 
of efferocytosis related proteins in hippocampus after CLP surgery eight days by western blot. (d) Expression of Rac1 (f) MFGE8, (g) LC3 ( ${ }^{*} p<0.01$, compared with sham group; $\# p<0.01$, compared with CLP group, one way ANOVA, $N=5$ ).

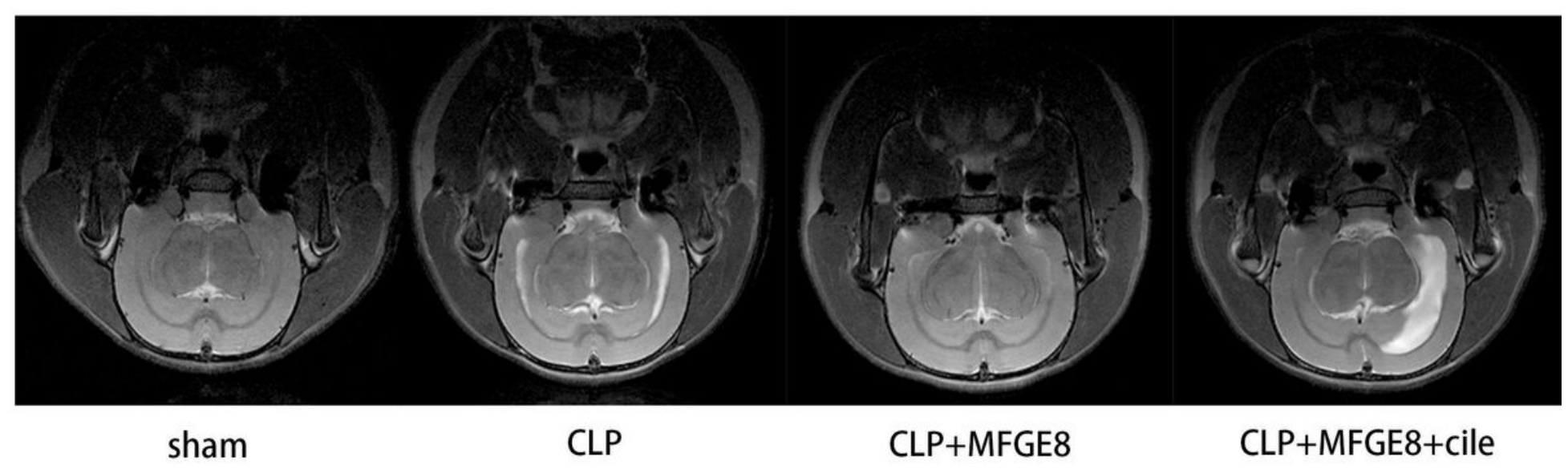

\section{Figure 3}

MRI tracking of structural damage in the hippocampus of rats among four groups.

Sepsis rats with encephalopathy underwent MRI scan, showing that a regional signal increase was produced in the hippocampus. However, this abnormal signals were reduced in CLP+MFGE8 group. In CLP+MFGE8+cilengitide group, the hyperintense focus in the region of hippocampus, and the range of damage areas were significantly large than that in CLP group. 


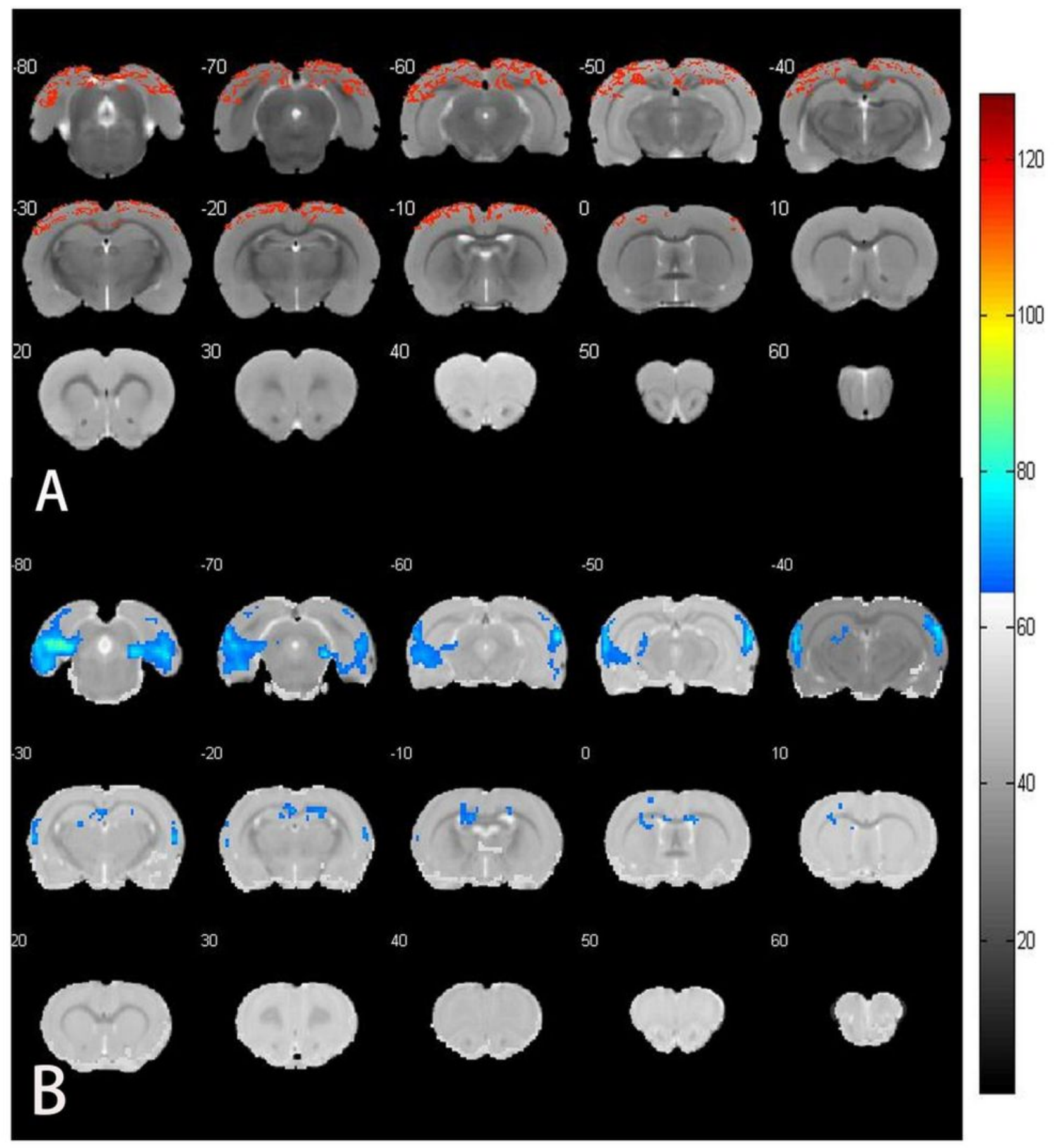

\section{Figure 4}

fMRI detected the functional changes between groups

(a) Differences of ALFF Between CLP group and CLP+MFGE8 group with whole-brain BOLD fRMI maps. The ALFF value of the bilateral sensory cortex, motor cortex and visual cortex were higher in SAE+MFGE8 group rats. (b) Differences of ALFF Between CLP group and CLP+MFGE8+cilengtide group. The ALFF 
value of auditory cortex, lateral entorhinal cortex (LEnt), perirhinal cortex (PRh), and dorsal hippocampal commissure (Dhc) were lower in SAE+MFGE8+cilengtide group rats.
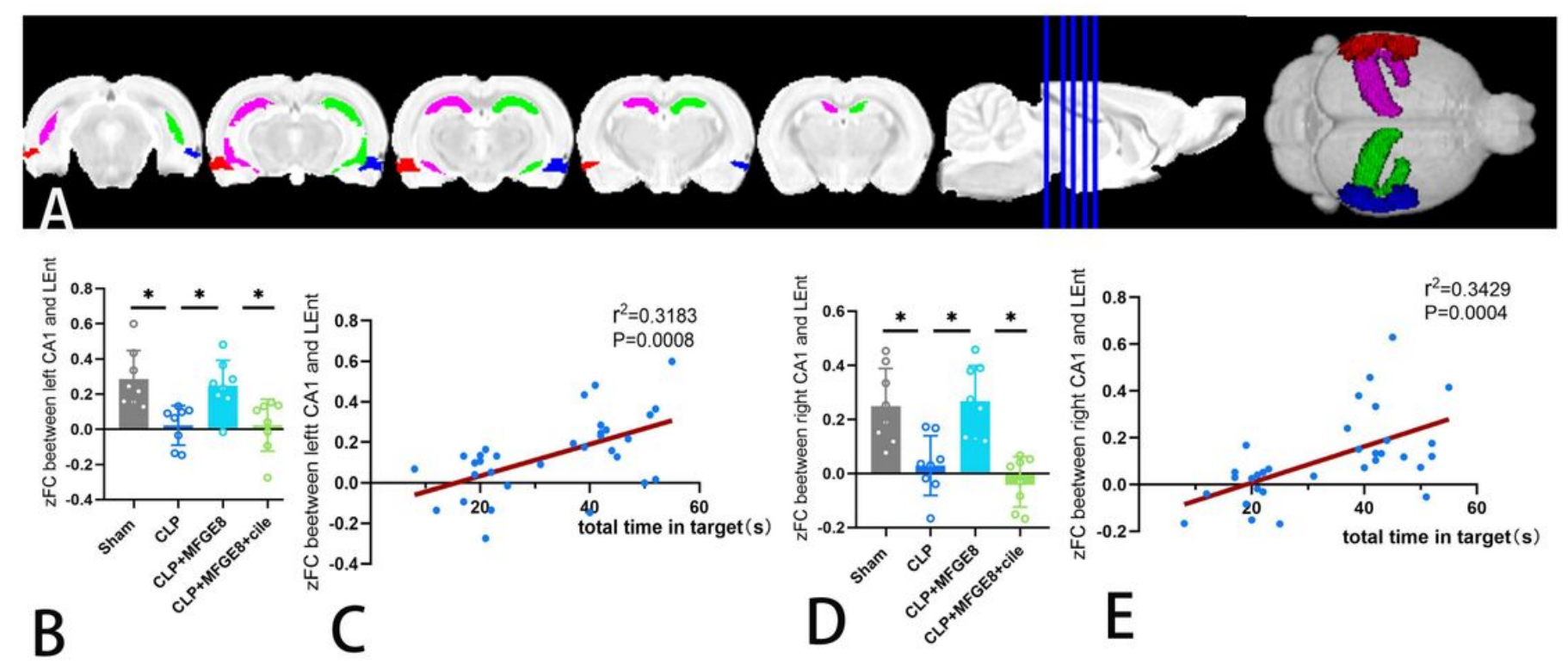

\section{Figure 5}

FC of CA1 and LEnt in cerebral hemisphere and the relationship between FC and behavior test result.

(a) ROls for functional connectivity analysis and relative location in rats' brain. left CA1 of hippocampus (pink area), right CA1 of hippocampus (green area), left LEnt (red area) and right LEnt (blue area). (b) The FC between the left CA1 and LEnt in four groups. $\left(F_{(3,28)}=7.779, p<0.05\right.$, ANOVA with Tukey's multiple comparisons test, ${ }^{*} p<0.01, N=8$ ). (c) FC between the left CA1 and LEnt was found positively correlated with total time in target quadrant in MWM test $\left(r^{2}=0.3183, p=0.0008\right)$. (d) The FC between the right CA1 and LEnt in four groups. $\left(\mathrm{F}_{(3,28)}=12.67, p<0.05\right.$, ANOVA, $\left.{ }^{\star} p<0.01, N=8\right)$. (e) $\mathrm{FC}$ in right CA1 and LEnt was also positively correlated with behavior test results. $\left(r^{2}=0.3429, p=0.0004\right)$.

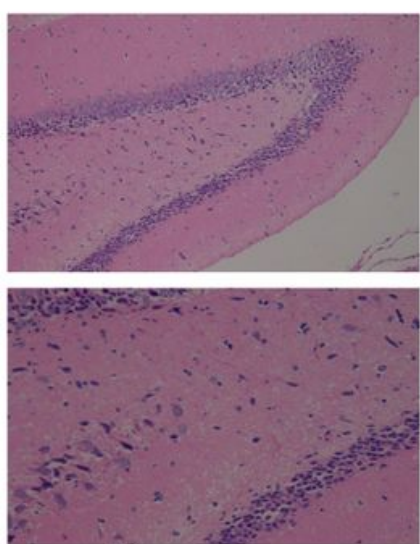

Sham
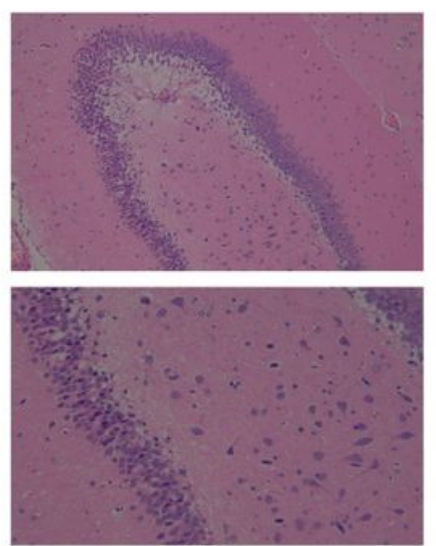

CLP
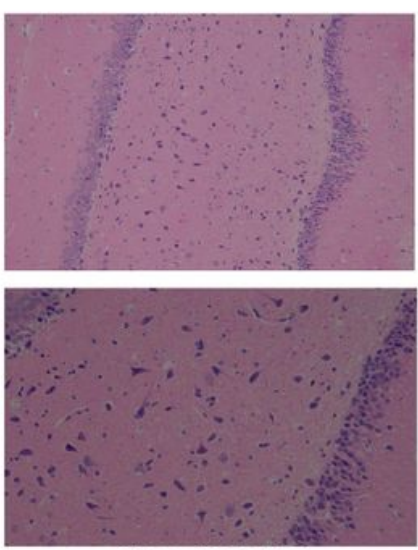

CLP+MFGE8

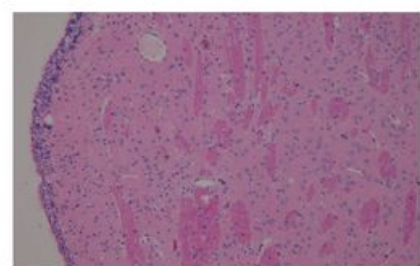

$\mathrm{x} 100$

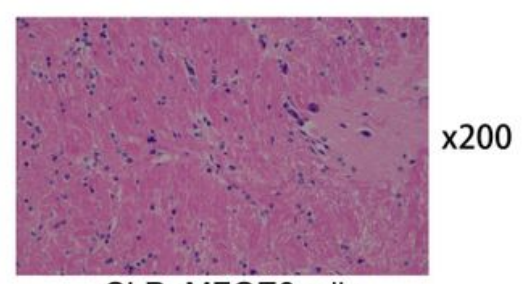

CLP+MFGE8+cile 


\section{Figure 6}

The pathological analysis of the brain sections stained with hematoxylin and eosin (H\&E). The pathological changes were focus on hippocampus, $(N=5)$. In sham group, pyramidal cells are arranged orderly in the hippocampus. In CLP group, cellular structure was disordered, and vacuole bodies appeared. In CLP+MFGE8 group, activated microglia accumulated in the white mater with homogeneous cytoplasmic staining. In CLP+MFGE8+cilengitide group, neuronal cells were lysis and cytoplasm edema, as well as eosin stain increased in the cytoplasm. 